\title{
Preclinical Testing of a Novel Thin Film Nitinol Flow-Diversion Stent in a Rabbit Elastase Aneurysm Model
}

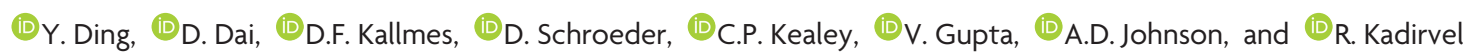

\begin{abstract}
BACKGROUND AND PURPOSE: Thin film nitinol can be processed to produce a thin microporous sheet with a low percentage of metal coverage $(<20 \%)$ and high pore attenuation $\left(\sim 70\right.$ pores $\left./ \mathrm{mm}^{2}\right)$ for flow diversion. We present in vivo results from the treatment of experimental rabbit aneurysms by using a thin film nitinol-based flow-diversion device.
\end{abstract}

MATERIALS AND METHODS: Nineteen aneurysms in the rabbit elastase aneurysm model were treated with a single thin film nitinol flow diverter. Devices were also placed over 17 lumbar arteries to model perianeurysmal branch arteries of the intracranial circulation. Angiography was performed at 2 weeks $(n=7)$, 1 month $(n=8)$, and 3 months $(n=4)$ immediately before sacrifice. Aneurysm occlusion was graded on a 3-point scale (grade I, complete occlusion; grade II, near-complete occlusion; grade III, incomplete occlusion). Toluidine blue staining was used for histologic evaluation. En face CD31 immunofluorescent staining was performed to quantify neck endothelialization.

RESULTS: Markedly reduced intra-aneurysmal flow was observed on angiography immediately after device placement in all aneurysms. Grade I or II occlusion was noted in 4 (57\%) aneurysms at 2-week, in 6 (75\%) aneurysms at 4-week, and in 3 (75\%) aneurysms at 12-week follow-up. All 17 lumbar arteries were patent. CD31 staining showed that $75 \% \pm 16 \%$ of the aneurysm neck region was endothelialized. Histopathology demonstrated incorporation of the thin film nitinol flow diverter into the vessel wall and no evidence of excessive neointimal hyperplasia.

CONCLUSIONS: In this rabbit model, the thin film nitinol flow diverter achieved high rates of aneurysm occlusion and promoted tissue in-growth and aneurysm neck healing, even early after implantation.

ABBREVIATION: TFN $=$ thin film nitinol

$\mathbf{F}$ low diverters are a relatively recent advancement in the endovascular treatment of intracranial aneurysms and have expanded the types of aneurysms addressable with endovascular techniques. ${ }^{1-5}$ Numerous different flow diverters have been approved in Europe, and several are either approved for use or under investigation in the United States. Each of the flow-diversion devices in current use is

Received June 9, 2015; accepted after revision August 12.

From the Department of Neurointerventional Radiology (Y.D., D.D., D.F.K., D.S., R.K.), Mayo Clinic, Rochester, Minnesota; and NeuroSigma Inc. (C.P.K., V.G., A.D.J.), Los Angeles, California.

This work was supported by NeuroSigma Inc. and a National Institutes of Health Grant (R41 NS074576).

Paper previously presented at: American Society of Neuroradiology Annual Meeting and the Foundation of the ASNR Symposium, April 25-30, 2015; Chicago, Illinois.

Please address correspondence to Yonghong Ding, MD, Department of Radiology, Mayo Clinic, 200 First St SW, Rochester, MN, 55905; e-mail: ding.yonghong@ mayo.edu

-- Indicates open access to non-subscribers at www.ajnr.org

http://dx.doi.org/10.3174/ajnr.A4568 constructed from braided metallic strands, typically nitinol, cobalt chromium, and/or platinum. These devices, while promising, have several relative disadvantages. Aneurysm occlusion may be delayed, precise placement may be challenging because of device shortening, $>1$ device is often required, and branch arteries covered by the device may undergo occlusion. ${ }^{6-8}$

Thin film nitinol (TFN) is a biomaterial produced in patterned sheets approximately $5 \mu \mathrm{m}$ thick by using techniques adapted from the microelectronics industry. Previous reports have demonstrated that TFN has unique mechanical properties, excellent biocompatibility, and a low profile that make it wellsuited for use in endovascular devices. ${ }^{9-11}$ Potential advantages of a flow-diverting stent based on TFN technology include the ability to fabricate devices with much higher pore densities and a lower percentage of metal coverage than is achieved with current-generation devices based on braided wire technology. The purpose of this study was to test a novel TFN-based flow-diverting stent in a rabbit model of saccular aneurysms. 

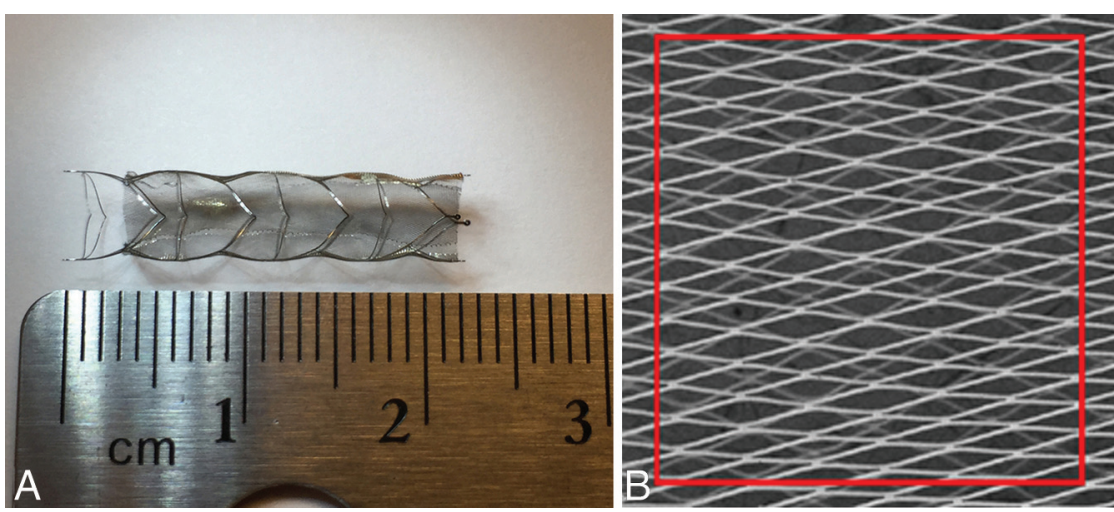

FIG 1. A, A prototype TFN flow diverter. $B$, The scanning electron microscopy image of TFN

\section{MATERIALS AND METHODS}

Aneurysms $(n=19)$ were created in New Zealand white rabbits. Our Institutional Animal Care and Use Committee approved all animal procedures. The detailed procedure for aneurysm creation has been described previously. ${ }^{12}$ Briefly, anesthesia was induced with an intramuscular injection of ketamine $(35 \mathrm{mg} / \mathrm{kg})$, xylazine $(6 \mathrm{mg} / \mathrm{kg})$, and acepromazine $(1.0$ $\mathrm{mg} / \mathrm{kg}$ ) and was maintained with $2.5 \%-3.0 \%$ isoflurane conveyed in $100 \%$ oxygen. Using a sterile technique, we exposed and ligated the right common carotid artery distally. A 1- to 2-mm bevelled arteriotomy was made, and a 5F AVANTI vascular sheath (Cordis, Miami Lakes, Florida) was advanced retrogradely in the right common carotid artery to a point approximately $3 \mathrm{~cm}$ cephalad to the origin of right common carotid artery. Fluoroscopy (Advantx; GE Healthcare, Milwaukee, Wisconsin) was performed by injection of contrast through the sheath retrogradely in the right common carotid artery, to identify the junction between the right common carotid artery and the subclavian and brachiocephalic arteries. A 3F Fogarty balloon (Baxter Healthcare, Irvine, California) was advanced through the sheath to the level of the origin of the right common carotid artery with fluoroscopic guidance and was inflated with iodinated contrast material. Porcine elastase (5.23 units per mg protein, $40.1 \mathrm{mg}$ protein/mL, approximately 200 units/mL; Worthington Biochemical, Lakewood, New Jersey) was incubated within the lumen of the right common carotid artery above the inflated balloon for 20 minutes, after which the balloon and sheath were removed and the right common carotid artery was ligated below the sheath entry site.

Three weeks after creation, patency of all the aneurysms and parent arteries was confirmed by DSA before TFN-device deployment. A 5F sheath was advanced on one side of the femoral artery via cutdown, followed by a 5F Envoy guiding catheter with 0.056 inch ID (Codman \& Shurtleff, Raynham, Massachusetts). A distal-access catheter with 0.044-inch ID (Concentric Medical, Mountain View, California) was advanced into the distal end of parent artery (right subclavian artery) over a 0.038-inch guidewire with a hydrophilic coating (Boston Scientific, Natick, Massachusetts) through the guide catheter.

Prototype TFN flow diverters were fabricated and provided for this study by NeuroSigma (Los Angeles, California). Detailed methods for the fabrication of TFN have been published previously. ${ }^{13-15}$ In brief, TFN is sputter-deposited on 4-inch silicon wafers by using a custom DC magnetron sputter system. Silicon wafers are micropatterned by using deep reactive ion etching before the sputterdeposition process. Following deposition, the TFN is removed from the silicon wafer and annealed at $500^{\circ} \mathrm{C}$. This process yields a cylindric TFN micromesh that is subsequently used to cover a laser-cut nitinol backbone stent (Fig 1). The red box outlines an area of $1 \mathrm{~mm}^{2}$. The pore attenuation and percentage of metal coverage of the TFN flow diverter were calculated from scanning electron microscope images of a device at full expansion. The number of pores in an area of $1 \mathrm{~mm}^{2}$ was counted from the scanning electron microscope image, and the percentage of metal coverage was calculated from the following formula: $1-$ Percent Metal Coverage of Stent Backbone. At full expansion, the TFN flow diverter had a pore attenuation of approximately 70 pores $/ \mathrm{mm}^{2}$ and a percentage of metal coverage of $<20 \%$. Results from in vitro and in vivo testing of devices constructed by using similar methods have been reported previously. ${ }^{9-11}$

The first TFN device $(4.5-\mathrm{mm}$ outside diameter $\times 12 \mathrm{~mm})$ was deployed across the aneurysm neck by pushing the device out of the distal access catheter with the 0.038 -inch guidewire with hydrophilic coating. The second device (4.5-mm outside diameter $\times 12 \mathrm{~mm}$ ) was deployed across a lumbar artery within the abdominal aorta. DSA was performed through the guide catheter immediately after deployment. No damage to the device occurred during deployment. Aspirin (10 mg/kg) and clopidogrel (10 mg/ $\mathrm{kg}$ ) were given daily 2 days before implantation and continued until 30 days after treatment.

Sacrifice was performed at 2 weeks $(n=7), 4$ weeks $(n=8)$, and 12 weeks $(n=4)$ after treatment. On the day of sacrifice, anesthesia was administered as a cocktail of ketamine $(74 \mathrm{mg} / \mathrm{kg})$, xylazine $(5 \mathrm{mg} / \mathrm{kg})$, and acepromazine $(1 \mathrm{mg} / \mathrm{kg})$. Surgical access of the left common femoral artery was achieved. DSA was performed for both the brachiocephalic trunk and abdominal aorta. Degrees of aneurysm occlusion immediately after device deployment and before sacrifice were graded on a 3-point scale based on DSA images, including grade I (complete flow cessation, no flow within the aneurysm), grade II (near-complete flow, $<10 \%$ residual flow), and grade III (incomplete occlusion, $\geq 10 \%$ residual flow). ${ }^{13}$ Patency of the parent and lumbar arteries (including stenosis or occlusion) was assessed from DSA. Immediately following angiography, the subjects were euthanized by using a lethal injection of pentobarbital. The aneurysm, stented parent artery, and the aorta were harvested and fixed in $10 \%$ formalin. Toluidine blue staining was performed to evaluate thrombus organization within the aneurysm and neointima coverage of aneurysm neck and the orifice of lumbar artery.

Gross pathology and en face CD31 immunofluorescent staining were performed on a subset of devices selected at random from each of the follow-up time points to quantify neck endothelialization ( 1 at 2 weeks, 3 at 4 weeks, 2 at 12 weeks). Wholemount immunofluorescent staining was performed by using an 
anti-CD31 antibody. The coverage percentage of endothelialized neointima across the neck was calculated by using the value of the neck area of endothelialization measured under the microscope and the whole neck area. Histopathology of explanted devices was performed on the device at each of the 3 time points by using plastic-section mounting and toluidine blue staining.

\section{RESULTS}

Mean aneurysm sizes (including aneurysm neck, width, and height) and angiographic outcomes from the 19 aneurysms are shown in the Table.

Grades I or II occlusion rates were noted in 57\% $(n=4)$ of aneurysms at the 2 -week follow-up time point (Fig $2 A-C$ ). At the 4-week time point, 6 (75\%) aneurysms had complete or nearcomplete occlusion (grades I or II) (Fig 3A-C). At the 12-week time point, 3 (75\%) aneurysms showed grades I or II occlusion. The distal parent artery was occluded in 1 aneurysm immediately after device deployment but reopened at the 3-month follow-up. Overall, grades I or II occlusion rates were achieved in 13 (68\%) of the 19 aneurysms. All other parent and lumbar arteries remained patent without stenosis (Figs $2 E-G$ and $3 E-G$ ).

For the 6 aneurysms with histologic processing, the average implant duration was 6.3 weeks. The mean neck orifice area was $6.3 \pm 2.5 \mathrm{~mm}^{2}$, and $75 \pm 16 \%$ of the aneurysm neck region was covered by endothelialized tissue at the time of sacrifice (Figs $2 D$ and $3 D$ ). Toluidine blue staining of aneurysms with explanted devices confirmed these findings, which included minimal neointimal hyperplasia and good incorporation of the TFN and support stent. Thrombus formation within the aneurysm was also indicated (Fig 4).

\section{DISCUSSION}

In this study, we demonstrated that a single TFN flow diverter could achieve high rates of complete or near-complete aneurysm occlusion as early as 2 weeks after implantation. Furthermore, rapid and near-complete endothelialization was noted across aneurysm necks, while branch arteries remained patent in all cases. All these results offer evidence that the TFN flow diverter holds substantial promise for clinical use.

Numerous flow-diverting devices have previously been tested in the elastase aneurysm model and have subsequently been applied clinically. ${ }^{16-22}$ Aneurysm occlusion, neointimal hyperplasia of the parent artery (stenosis or occlusion), distal parent artery emboli, and patency of the branch artery can be assessed in this aneurysm model. Compared with current flow-diverter devices, the NeuroSigma TFN flow diverter achieves a very high pore attenuation while, at the same time, allowing a low percentage of metal coverage. Thus, very small distances are needed for endothelial cells to cross between structural elements of the TFN.

This study has several limitations. The number of subjects at each time point was relatively small, and the duration of implantation was limited. The aneurysms in this study were small, even though the mean height of the aneurysm was approximately $10 \mathrm{~mm}$. The longest time point for follow-up was only 3 months in this study. Finally, the morphology of
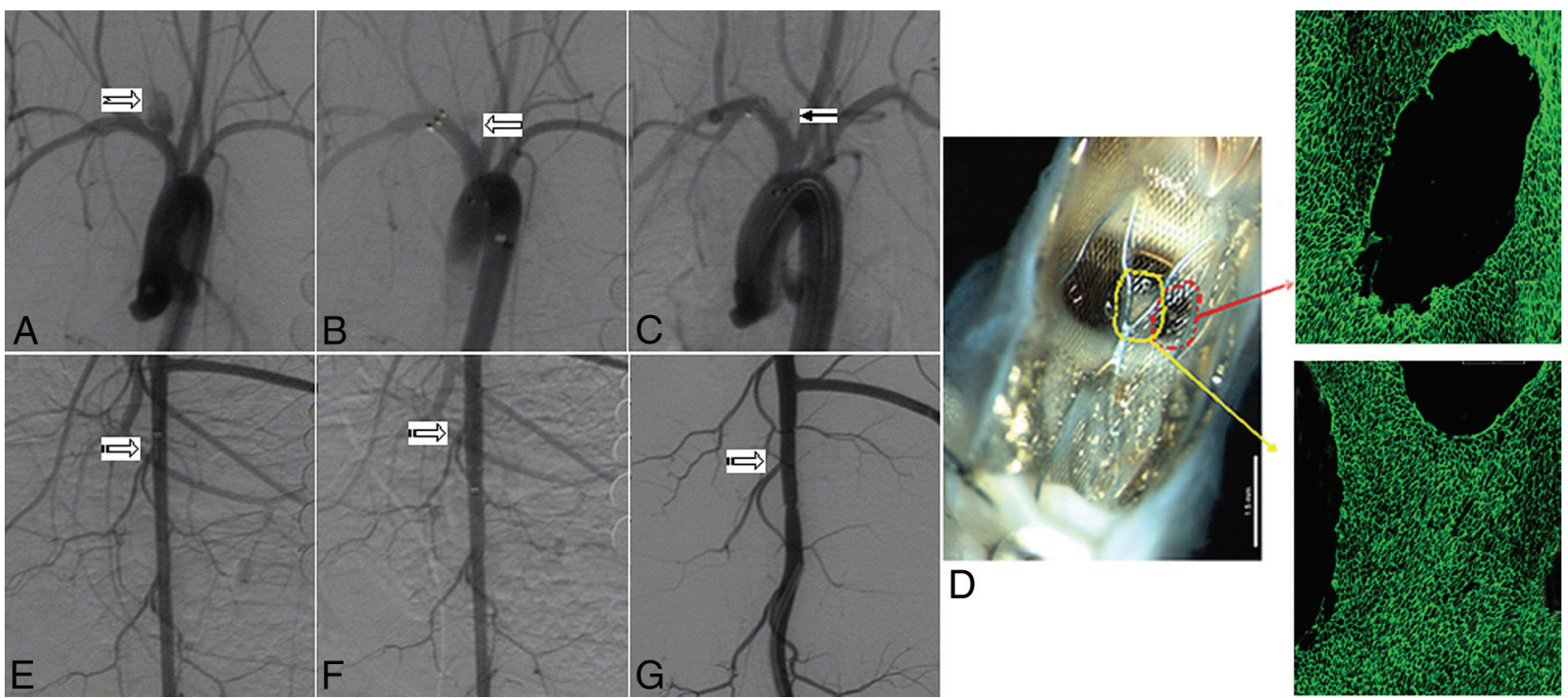

FIG 2. A, Digital subtraction angiogram shows the aneurysm before treatment (notched right arrow). $B$, DSA image immediately after device deployment shows blood flow reduction in the aneurysm (left block arrow). C, DSA image at 2 weeks of deployment shows near-complete aneurysm occlusion (grade II) (left arrow). D, Gross pathology along with en face CD 31 immunofluorescent staining (original magnification water lens $20 \times$ ) shows that $46 \%$ of aneurysm neck area is covered by endothelialized tissue (red and yellow arrows). E, DSA image shows the lumbar arteries before deployment in the abdominal aorta (striped right arrow). F, DSA image immediately after device deployment shows patent lumbar arteries (striped right arrow). G, DSA image at 2 weeks of deployment shows that the lumbar arteries remain patent (striped right arrow). 

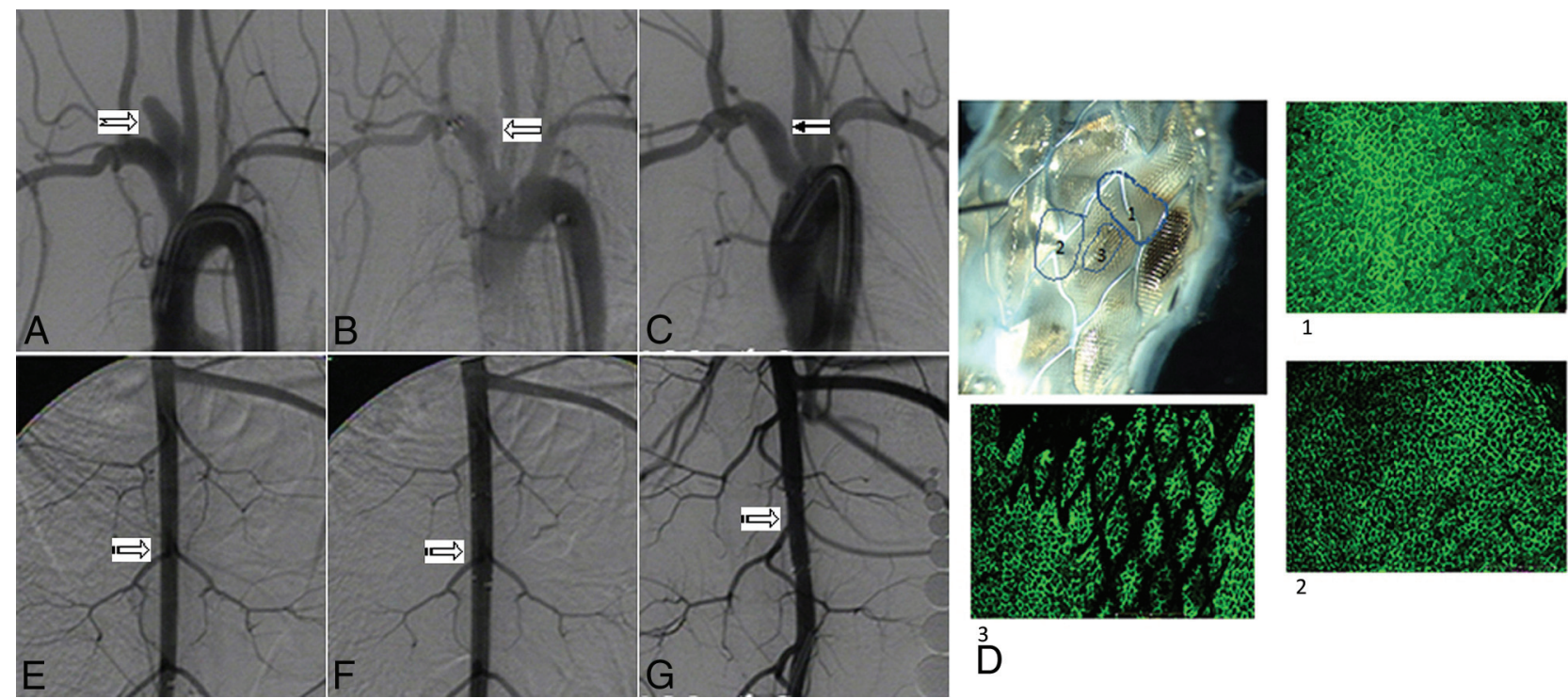

3

FIG 3. $A$, Digital subtraction angiogram shows the aneurysm before treatment (notched right arrow). $B$, DSA image immediately after device deployment shows significant blood flow reduction in the aneurysm (left block arrow). C, DSA image at 4 weeks of deployment shows complete aneurysm occlusion (grade I) (left arrow). D, Gross pathology along with en face CD 31 immunofluorescent staining (original magnification water lens $20 \times$ ) shows that $89 \%$ of the aneurysm neck area was covered by endothelialized tissue (areas 1, 2, and 3). E, DSA image shows the lumbar arteries before deployment in the abdominal aorta (striped right arrow). F, DSA image immediately after device deployment shows patent lumbar arteries (striped right arrow). G, DSA image at 4 weeks of deployment shows that the lumbar arteries remain patent (striped right arrow).

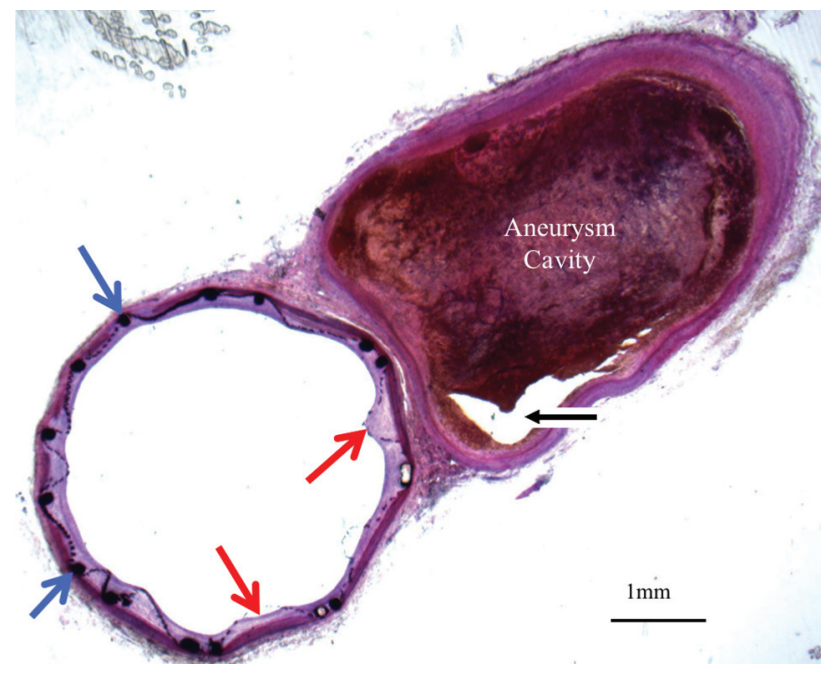

FIG 4. Histologic section (toluidine blue staining, original magnification $1.25 \times$ ) of the TFN flow diverter explanted after 4 weeks. This axial image demonstrates minimal neointimal hyperplasia deep to the TFN (red arrows) and support stent (blue arrows). The aneurysm cavity is partially filled with thrombus in various stages of organization. A small part of the aneurysm lumen is empty (black arrow).

the aneurysms does not provide the range expected clinically, and the tortuosity of the carotid siphon and other vessel territories in humans may cause substantial challenges in achieving adequate wall apposition of the device.

\section{CONCLUSIONS}

In this rabbit model, the TFN devices achieved high rates of acute angiographic occlusion. Vessel branches covered by the devices remained patent. High degrees of endothelialization across the aneurysm neck were achieved, which indicates that in this model, the TFN can promote tissue in-growth and aneurysm neck healing.
Disclosures: Yonghong Ding—RELATED: Grant: This work was partially supported by a National Institutes of Health Small Business Technology Transfer (STTR) grant (1R41NS074576). David F. Kallmes—RELATED: Grant: NeuroSigma, ${ }^{\star}$ Comments: National Institutes of Health Small Business Innovation Research (SBIR) grant subcontract; UNRELATED: Board Membership: GE Healthcare (cost-effectiveness board); Consultancy: ev3/Covidien/Medtronic, Comments: planning and implementing clinical trials; Grants/Grants Pending: MicroVention, ${ }^{*}$ Sequent, ${ }^{*}$ SurModics, ${ }^{*}$ Codman, ${ }^{\star}$ ev3/Covidien/Medtronic, ${ }^{*}$ Comments: preclinical and clinical research; $R$ Royalties: University of Virginia Patent Foundation (Spine Fusion); Travel/Accommodations/Meeting Expenses Unrelated to Activities Listed: ev3/Covidien/Medtronic, * Comments: travel to the FDA panel meeting. Colin P. Kealey-RELATED: The work was partially funded by a STTR grant from the National Institutes of Health (IR4INS074576)*; UNRELATED: Employment: NeuroSigma (full-time employee); Patents (planned, pending or issued): I am a coinventor on patents related to this technology that are owned and/or licensed by NeuroSigma*; Stock/Stock Options: I have stock options in NeuroSigma. Vikas Gupta—RELATED: Grant: National Institutes of Health *; Consulting Fee or Honorarium: NeuroSigma, Comments: Work during May 2014 to March 2015 was performed as an independent consultant. Since April 2015, the work has been performed as full-time employee; Support for Travel to Meetings for the Study or Other Purposes: NeuroSigma; UNRELATED: Consultancy: NeuroSigma; Employment: NeuroSigma; Stock/Stock Options: NeuroSigma; Travel/Accommodations/Meeting Expenses Unrelated to Activities Listed: NeuroSigma. Alfred David Johnson-RELATED: Grant: National Institutes of Health (IR41NS074576)*; Support for Travel to Meetings for the Study or Other Purposes: Travel expenses were paid for experiments at the Mayo Clinic by NeuroSigma; UNRELATED: Other: salary from TiNi Alloy Company for work unrelated to the submitted work. Ramanathan Kadirvel—RELATED: Grant: National Institutes of Health (NS076491).* *Money paid to the institution.

\section{REFERENCES}

1. Kallmes DF, Hanel R, Lopes D, et al. International retrospective study of the Pipeline embolization device: a multicenter aneurysm treatment study. AJNR Am J Neuroradiol 2015;36:108-15 CrossRef Medline

2. Saatci I, Yavuz K, Ozer C, et al. Treatment of intracranial aneurysms using the Pipeline flow-diverter embolization device: a single-center experience with long-term follow-up results. AJNR Am J Neuroradiol 2012;33:1436-46 CrossRef Medline

3. Berge J, Biondi A, Machi P, et al. Flow-diverter Silk stent for the treatment of intracranial aneurysms: 1-year follow-up in a multicenter study. AJNR Am J Neuroradiol 2012;33:1150-55 CrossRef Medline 
4. Lubicz B, Van der Elst O, Collignon L, et al. Silk flow-diverter stent for the treatment of intracranial aneurysms: a series of 58 patients with emphasis on long-term results. AJNR Am J Neuroradiol 2015; 36:542-46 CrossRef Medline

5. Briganti F, Leone G, Marseglia M, et al. p64 flow modulation device in the treatment of intracranial aneurysms: initial experience and technical aspects. J Neurointerv Surg 2015 Apr 20. [Epub ahead of print] CrossRef Medline

6. Tan LA, Keigher KM, Munich SA, et al. Thromboembolic complications with Pipeline embolization device placement: impact of procedure time, number of stents and pre-procedure $\mathrm{P} 2 \mathrm{Y} 12$ reaction unit (PRU) value. J Neurointervent Surg 2015;7:217-21 CrossRef Medline

7. Cirillo L, Leonardi M, Dall'olio M, et al. Complications in the treatment of intracranial aneurysms with Silk stents: an analysis of $\mathbf{3 0}$ consecutive patients. Interv Neuroradiol 2012;18:413-25 Medline

8. De Vries JD, Boogaarts J, Van Norden AV, et al. New generation of flow diverter (Surpass) for unruptured intracranial aneurysms: a prospective single-center study in 37 patients. Stroke 2013;44: 1567-77 CrossRef Medline

9. Shayan M, Chun Y. An overview of thin film nitinol endovascular devices. Acta Biomater 2015;21:20-34 CrossRef Medline

10. Kealey CP, Chun YJ, Viñuela FE, et al. In vitro and in vivo testing of a novel, hyperelastic thin film nitinol flow diversion stent. J Biomed Mater Res B Appl Biomater 2012;100:718-25 CrossRef Medline

11. Kealey CP, Whelan SA, Chun YJ, et al. In vitro hemocompatibility of thin film nitinol in stenotic flow conditions. Biomaterials 2010;31: 8864-71 CrossRef Medline

12. Ding YH, Dai D, Kadirvel R, et al. Five-year follow-up in elastaseinduced aneurysms in rabbits. AJNR Am J Neuroradiol 2010;31: 1236-39 CrossRef Medline

13. Gupta V, Johnson AD, Martynov V, et al. Nitinol thin film threedimensional devices: fabrication and applications. In: Pelton AR, Duerig T, eds. SMST-2003: Proceedings. Materials Park: ASM International 2004;639-50
14. Mohanchandra KP, Ho KK, Carman GP. Compositional uniformity in sputter-deposited NiTi shape memory alloy thin films. Materials Lett 2008;62:3481-83 CrossRef

15. Chun YJ, Levi DS, Mohanchandra KP, et al. Novel micro-patterning processes for thin film NiTi vascular devices. Smart Materials and Structures 2010;19:105021 CrossRef

16. Brinjikji W, Lanzino G, Cloft HJ, et al. Patency of the posterior communicating artery after flow diversion treatment of internal carotid artery aneurysms. Clin Neurol Neurosurg 2014;120:84-8 CrossRef Medline

17. Kallmes DF, Ding YH, Dai D, et al. A new endoluminal, flow-disrupting device for treatment of saccular aneurysms. Stroke 2007;38: 2346-52 CrossRef Medline

18. Kallmes DF, Ding YH, Dai D, et al. A second-generation, endoluminal, flow-disrupting device for treatment of saccular aneurysms. AJNR Am J Neuroradiol 2009;30:1153-58 CrossRef Medline

19. Simgen A, Ley D, Roth C, et al. Evaluation of a newly designed flow diverter for the treatment of intracranial aneurysms in an elastaseinduced aneurysm model, in New Zealand white rabbits. Neuroradiology 2014;56:129-37 CrossRef Medline

20. Struffert T, Ott S, Kowarschik M, et al. Measurement of quantifiable parameters by time-density curves in the elastase-induced aneurysm model: first results in the comparison of a flow diverter and a conventional aneurysm stent. Eur Radiol 2013;23:521-27 CrossRef Medline

21. Ionita CN, Natarajan SK, Wang W, et al. Evaluation of a secondgeneration self-expanding variable-porosity flow diverter in a rabbit elastase aneurysm model. AJNR Am J Neuroradiol 2011;32:1399407 CrossRef Medline

22. Sadasivan C, Cesar L, Seong J, et al. An original flow diversion device for the treatment of intracranial aneurysms: evaluation in the rabbit elastase-induced model. Stroke 2009;40:952-58 CrossRef Medline 